\title{
Conceptualización y evaluación de la impulsividad en adolescentes: una revisión sistemática*
}

\author{
Conceptualization and Assessment of Impulsivity \\ in Adolescents: a Systematic Review
}

Recibido: 1 de febrero de 2015 | Revisado: 10 de mayo de 2015 | Aceptado: 20 de julio de 2015

\author{
DiANA RIAÑO-HERNÁNDEZ ** \\ AleJANDRo GUILLEN RiQuelme \\ GuAlBerto Buela-CASAL \\ Universidad de Granada, Granada, España
}

doi:10.11144/Javeriana.upsy14-3.ceia

Para citar este artículo: Riaño-Hernández, D., Guillen, A., \& Buela-Casal, G. (2015). Conceptualización y evaluación de la impulsividad en adolescentes: una revisión sistemática. Universitas Psychologica, 14(3), 1077-1090. http://dx.doi. org/10.11144/Javeriana.upsy14-3.ceia

\section{* Artículo de revisión}

** Centro de Investigación Mente, Cerebro y Comportamiento (CIMCYC), Universidad de Granada. Cercado Bajo de Cartuja, Granada, España. Fundación Universitaria Konrad Lorenz, Bogotá, Colombia.

Nota. Debido a que se han incluido 108 artículos en la revisión, la lista completa de referencias se enviará solicitando una copia al autor de contacto. Correo electrónico: dianarianoh@gmail.com

\section{RES UMEN}

La impulsividad es un constructo ampliamente estudiado y se ha caracterizado por la falta de consenso entre los autores, sobre su definición, características y formas de evaluación, con la consecuente complejidad actual. Se realizó la revisión sistemática sobre impulsividad en adolescentes, con el objetivo de identificar las diferentes definiciones, analizar las medidas que son usadas para evaluarla y puntualizar los diversos rasgos psicológicos con los cuales se relaciona, en la investigación. La recopilación de artículos se hizo por medio de las bases de datos Web of Knowledge, Scopus, PsycInfo, Scielo, Redalyc, Google Escolar y Cochrane, donde se seleccionaron 3457 artículos, de los cuales se revisaron 108. Los resultados ponen de manifiesto las definiciones de impulsividad, los participantes, las medidas de evaluación y la relación con otras variables. Se concluye que (a) la impulsividad es un constructo con múltiples definiciones, (b) se identificó el MFFT como medida más usada para evaluarlo y (c) se encontró que se ha investigado su relación en temas como problemas de conducta, medidas cognitivas, agresión y aspectos familiares. Palabras clave

revisión; impulsividad; adolescentes

\footnotetext{
A B S T R A C T

Impulsivity is a widely studied construct and has been characterized by a lack of consensus among authors about its definition, characteristics and assessment. This has provoked that impulsivity can be seen as a complex construct. We have conducted a systematic review of impulsivity in adolescents, in order to identify the different definitions, analyze the measures that are used to assess impulsivity and point out the different features which are related to the research on impulsivity. The literature was collected in an exhaustive search from several databases: Web of Knowledge, Scopus, PsycINFO, Scielo, Redalyc, Google School and Cochrane, where a set of 3,457 papers was selected. From this initial set, we reviewed 108 papers. Our results showed the definitions of impulsivity, the population, the evaluation measures and the relationship with other variables. We concluded that, (a) impulsivity has multiple definitions, (b) the most used measure to assess the construct is the MFFT and (c) we found that impulsivity has been research on topics such as behavioral problems, cognitive measures, assault and family issues. Keywords review; impulsivity; adolescents
} 
La impulsividad es una temática ampliamente estudiada que presenta un creciente interés. En la última década, se han generado diferentes revisiones sistemáticas sobre la relación de impulsividad con otros aspectos como: agresión (Poythress \& Hall, 2011), consumo de sustancias (Coskunpinar \& Cyders, 2013; Grant \& Chamberlain, 2014), déficit de atención (Cabral, 2006; Winstanley, Eagle, \& Robbins, 2006), depresión (Saddichha $\&$ Schuetz, 2014), trastorno bipolar (Najt et al., 2007), esquizofrenia (Ouzir, 2013), psicosis (Bjørkly, 2013), trastornos alimentarios (Fischer, Smith, \& Cyders, 2008) y variables neuropsicológicas (Bari \& Robbins, 2013), entre otras. Estas revisiones no se centran en el abordaje de estudios con población adolescente, y su principal interés no es la impulsividad sino la relación de esta con otros aspectos.

El estudio de la impulsividad se ha caracterizado por la falta de consenso entre los autores sobre su definición, características, dimensiones y formas de evaluación; consecuentemente, en la actualidad es un constructo complejo que se aborda desde varias estrategias (Buelow \& Suhr, 2009; Fields, Sabet, \& Reynolds, 2013; Janssen et al., 2015; Leshem \& Glicksohn, 2012; Whiteside \& Lynam, 2001). A continuación, se plantea y se contextualiza cada uno de estos aspectos.

\section{Definición de impulsividad}

Varios autores (Buelow \& Suhr, 2009; Donohew et al., 2000; Dougherty et al., 2003; Gullo \& Dawe, 2008; Khodarahimi, 2013; Martínez-Loredo, Fernández-Hermida, Fernández-Artamendi, Carballo, \& García-Rodríguez, 2015; Romer, 2010; Thompson, Whitmore, Raymond, \& Crowley, 2006) señalan que el constructo de impulsividad es multidimensional o multifacético, reconociendo la dificultad en cuanto a su aproximación y definición.

En las publicaciones sobre el tema se encuentra una gran gama de definiciones, tales como la falta de inhibición de respuestas y procesamiento rápido de la información (Barratt,1994), la tendencia a vivir el momento sin tener en cuenta las consecuencias en el futuro, actuar sin pensar (Castellani \& Rugle, 1995; Ghahremani et al., 2013; Kaltiala-
Heino, Rissanen, Rimpelä, \& Rantanen, 2003), la respuesta sin tener una evaluación adecuada del contexto, la dificultad en retardar las recompensas y la toma de decisiones rápidas (Claes, Vertommen, \& Braspenning, 2000; Dougherty et al., 2003; Reynolds, Penfold, \& Patak, 2008) y la falta de autocontrol (Brook \& Boaz, 2005). También aparece definida como una preferencia hacia pequeñas y prontas recompensas sobre recompensas más grandes y demoradas, generando respuestas prematuras que evidencian la falta de inhibición (Dougherty et al., 2003), o como un estilo cognitivo que se caracteriza por la falta de reflexividad y se centra en observar las respuestas en cuanto a su duración y errores cometidos. Así pues la gente impulsiva tendería a un estilo de respuesta caracterizado por responder rápido y sin demasiado análisis, y que se genera cuando los individuos se enfrentan a problemas que cuentan con varias opciones de solución, pero solo una de ellas es la correcta, por esta razón, se relaciona con procesos de evaluación y producción de alternativas (Buela-Casal, Carretero-Dios, \& Santos-Roig, 2001; Oas, 1984; Plutchik \& Van Praag, 1995).

\section{Dimensiones de la impulsividad}

La falta de una definición precisa y aceptada de forma general ha llevado a conformar diferentes dimensiones que acompañan la conceptualización de la impulsividad. DeWit (2009) y Whiteside y Lynam (2001) aclaran el constructo proponiendo un modelo identificado por cuatro componentes: urgencia negativa (tendencia a actuar impulsivamente cuando se experimenta una situación negativa); falta de perseverancia (tendencia a rendirse al enfrentar el aburrimiento); falta de premeditación (tendencia a actuar sin tener en cuenta las consecuencias) y búsqueda de sensaciones (tendencia a buscar actividades que resulten excitantes).

Por otra parte, se identifican tres rasgos de la impulsividad: un componente motor (actuar sin pensar), un componente atencional (inestabilidad cognitiva y atencional) y, el último, un componente de no planificación (falta de planificación) (Gilbert et al., 2011; Patton, Stanford, \& Barrat, 1995). 
Otros componentes mencionados por Letzring, Block y Funder (2005) hacen referencia a la impulsividad como control del ego caracterizado por un continuo de poco control a mucho control, donde los individuos con poco control tienen dificultades para retrasar las recompensas, son espontáneos y se distraen fácilmente.

El modelo sugerido por Mathias, Marsh-Richard y Dougherty (2008) distingue tres tipos diferentes de impulsividad; (a) la iniciación de respuesta como acciones espontáneas que no son planeadas; (b) la inhibición de la respuesta como la dificultad al inhibir respuestas rápidas y acciones no planeadas una vez se ha iniciado la acción y (c) la sensibilidad a las consecuencias, como el fracaso para tolerar retrasos en las recompensas. Reynolds et al. (2008) conceptualizan la impulsividad por medio de tres dimensiones separadas que incluyen toma de decisiones, inatención y desinhibición. Por otra parte, Buela-Casal, Carretero-Dios, Santos-Roig y Bermúdez (2003) proponen dos dimensiones, la primera referente al estilo de Impulsividad-Reflexividad en el que las personas que responden con gran número de errores y corto tiempo son impulsivos y otras con un bajo número de errores y alto tiempo son reflexivos. La segunda dimensión indica el nivel de competencia, algunas personas responden en corto tiempo y con un bajo número de errores (eficientes) o responden con un alto número de errores en un periodo de tiempo largo (ineficientes). Dickman (1990) plantea dos componentes: la impulsividad funcional y disfuncional. La primera está relacionada con la tendencia a responder y reaccionar de forma rápida que en ocasiones resulta beneficiosa y lleva a consecuencias positivas; la segunda estaría relacionada con la obtención de consecuencias negativas, siendo estas acciones rápidas perjudiciales para la persona.

\section{Evaluación de la impulsividad}

La diversidad de definiciones y componentes de la impulsividad han ocasionado la construcción de diversos instrumentos de evaluación. Estos se pueden clasificar en dos tipos (a) cuestionarios y autorregistros y (b) medidas conductuales o experimentales (Carver \& White, 1994; Derryberry \&
Reed, 2002; Glenn \& Klonsky, 2010; Khurana et al., 2012; Rachlin, 2004; Richards, Zhang, Mitchell, \& de Wit, 1999). En el desarrollo de la investigación, es muy frecuente emplear conjuntamente ambos tipos de instrumentos, esto es necesario para rastrear la complejidad del constructo, sin embargo, el uso de diferentes instrumentos dificulta comparar los resultados (Doughert et al., 2009; Gorlyn, Keilp, Tryon, \& Mann, 2005; Reynolds, Ortengren, Richards, \& de Wit, 2006).

Los resultados de la mayoría de estudios que usan varios instrumentos de evaluación de la impulsividad ponen de manifiesto correlaciones bajas, no significativas o inexistentes, lo que sugiere la falta de congruencia (cuestionarios frente a medidas conductuales), cuando se comparan los métodos de evaluación (Dougherty et al., 2009; Heyman \& Gibb, 2006; Krishnan-Sarin et al., 2007; Thompson et al., 2006; Wittmann \& Paulus, 2008). No obstante, se argumenta que esa falta de acuerdo no es tan evidente cuando las medidas que se están usando comparten características similares (Carrillo-DeLa-Peña, Otero, \& Romero, 1993).

Respecto a las medidas conductuales, Mathias et al. (2008) resaltan su objetividad como método para evaluar la impulsividad, ya que son menos propensas al engaño, no están mediadas por procesos de interpretación de la propia conducta, no dependen del recuerdo de circunstancias y es más difícil que los individuos tergiversen deliberadamente sus respuestas.

\section{Relación con otros constructos psicológicos}

La discusión y confusión sobre el concepto de impulsividad no solamente aborda la variabilidad en cuanto a su definición y evaluación, sino que abarca los constructos psicológicos con los cuales se relaciona la impulsividad (agresividad, adicción, obesidad, ansiedad, depresión, toma de decisiones, intentos de suicidio, etc.). En muchos estudios, se aborda la impulsividad como un constructo que se deriva o es subyacente a otros y no como independiente.

Se tiene en cuenta que si no existe claridad en la conceptualización y los métodos de evaluación 
de la impulsividad, los resultados de las investigaciones que la relacionan con otros rasgos psicológicos serían poco precisos. De aquí la importancia de producir estudios donde el objetivo principal sea identificar y aclarar la impulsividad y no solo abordarla como una característica más que se relaciona con otros rasgos. Esta revisión sistemática tiene como objetivo exponer la forma en que se estudia el constructo de impulsividad en adolescentes, identificando las diferentes definiciones de impulsividad, analizando las medidas que son usadas para evaluar impulsividad en adolescentes y puntualizando los diferentes rasgos psicológicos con las cuales se relaciona en las investigaciones la impulsividad.

\section{Método}

\section{Recopilación de datos}

Los estudios fueron obtenidos a través de las siguientes bases de datos: Web of Knowledge, Scopus,
PsycInfo, Scielo, Redalyc, Google Escolar y Cochrane. El intervalo de tiempo especificado fue de 1995 a 2014, ya que según la base de datos de Scopus, en 1995 es cuando empieza a aumentar el número de publicaciones sobre Impulsividad. Los términos de búsqueda fueron: Impulsivity AND adolescents; Impulsiv* AND adolesc*/teen*. El procedimiento se complementó mediante una búsqueda manual en las listas de referencias de los artículos, para detectar documentos adicionales que fueran pertinentes.

\section{Criterios de inclusión y exclusión}

Los criterios de inclusión fueron estudios de 1995 a 2014 realizados con adolescentes de 12 a 18 años de edad, en idioma español, inglés o portugués que desarrollaran evaluación del constructo de impulsividad. Solo se incluyeron artículos, ya que interesaba recopilar toda la información necesaria en la codificación de variables, por lo cual no se incluyeron documentos como conferencias, tesis o informes. Debido a que el interés principal fue elegir

TABLA 1

Variables extraídas

\begin{tabular}{ll}
\hline Datos generales & Autores, año, título, revista, palabras clave \\
\hline Impulsividad & $\begin{array}{l}\text { Definición de impulsividad, identificación de complejidad del constructo, identificación de } \\
\text { dimensiones del constructo }\end{array}$ \\
\hline Participantes & Media de edad, país de la muestra \\
\hline Instrumentos & $\begin{array}{l}\text { Instrumentos de impulsividad, si el instrumento estaba adaptado o no, otros constructos } \\
\text { evaluados }\end{array}$ \\
\hline Resultados & Relación de la impulsividad con otros constructos \\
\hline
\end{tabular}

Fuente: elaboración propia

Total de referencias recuperadas $n=3.457$

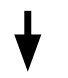

Artículos seleccionados luego de lectura del título $n=348$

$\downarrow$

Total seleccionados luego de leer el resumen $n=177$

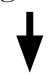

Total seleccionados luego de lectura completa $n=108$

Figura 1. Proceso de selección documentos.

Fuente: elaboración propia 
artículos que se centraran en la evaluación de la impulsividad en adolescentes, no se consideraron revisiones sistemáticas, ni documentos relacionados con farmacología ni experimentos con animales.

\section{Codificación de variables}

Las variables codificadas o los datos extraídos fueron agrupados en datos generales, impulsividad, participantes, instrumentos y resultados. En la Tabla 1 se detallan las variables que componen cada una de estas categorías.

\section{Depuración de artículos}

La depuración se llevó a cabo siguiendo los parámetros de Perestelo-Pérez (2013). El análisis de estudios para la elección de los documentos definitivos para la revisión se llevó a cabo primero examinando los títulos, luego los resúmenes y finalmente el texto completo (Figura 1).

\section{Resultados}

\section{Definición del constructo}

Se identificó que dentro de los 108 documentos revisados, en 16 no se incluye una definición de impulsividad, en 17 se reconoce que el constructo de impulsividad comprende una definición compleja, mientras que en 12 se define la impulsividad como una característica de otro constructo psicológico y en 63 se define conceptualmente el constructo de impulsividad. Estas definiciones se muestran en la Tabla 2.

\section{Participantes}

Dentro de los 108 documentos revisados, 79 ponen de manifiesto la procedencia de los participantes con los que habían realizado la evaluación de impulsividad con adolescentes, a saber: la mayor parte de los participantes evaluados eran europeos con 30 artículos; seguido por Estados Unidos con 27 artículos, se identificaron 15 artículos que hicieron la evaluación con participantes de habla hispana en países como México, Colombia, España y Chile, y finalmente, 7 estudios elaborados con participantes en Asia. La media aproximada para la edad fue de 15.3 con un intervalo de 12 a 18 años.

\section{Evaluación de impulsividad}

Se identificaron 29 instrumentos para evaluar impulsividad en adolescentes, dentro de los cuales 10 artículos especificaban que habían hecho uso de instrumentos adaptados. De estos 29 dispositivos de evaluación, 14 eran escalas o cuestionarios y 15 eran instrumentos de medidas conductuales. En la Tabla 3, se muestra el resumen de los 11 instrumentos más usados en los artículos, especificando la cantidad, la media del alfa de Cronbach, la descripción del instrumento y su nombre. Además, hay diversos instrumentos empleados solo una vez como medidas de evaluación conductuales, a saber: Delay Discounting Task, HV Haptic-Visual Test, Measure of Impulsive Decision Making, The ArrowDot Test, Temporal Discounting, Kirby Delay Discounting Measure, Bender Gestalt Test Draw-A-Person y Test Experiential Discounting Task. Dentro de los cuestionarios se identificaron: Internal Restlessness Scale, Dickman's Impulsivity Inventory, Behavior Rating Scale of Impulsivity, Impulsive Behavior Checklist, General Temperament Survey, The Stress Management, Hirschfield Scale, The IRS y Cuestionario Revisado de Resolución de Problemas.

\section{Relación de impulsividad con otros constructos}

Se encuentra que todos los artículos relacionan la impulsividad con algún otro aspecto. En la Tabla 4 se muestran estos aspectos. Por otra parte, se distinguen con una frecuencia de 1 , los aspectos como acupuntura, regulación emocional, problemas de sueño y búsqueda de sensaciones.

\section{Discusión}

El objetivo de esta revisión sistemática fue exponer la forma como se estudia el constructo de impulsividad en adolescentes: identificando las diferentes 


\section{TABLA 2}

Definiciones de impulsividad

\begin{tabular}{|c|c|c|}
\hline Definición de impulsividad & Autores relacionados & $\begin{array}{l}\text { Número } \\
\text { de } \\
\text { artículos }\end{array}$ \\
\hline $\begin{array}{l}\text { Déficit en el procesamiento cognitivo como una falla en } \\
\text { la inhibición de respuesta, un rápido procesamiento de } \\
\text { información, dificultad en retardar las recompensas, ten- } \\
\text { dencia a vivir el momento, no pensar antes de actuar. }\end{array}$ & (Barratt, 1994) & 5 \\
\hline $\begin{array}{l}\text { Se define por tres características: (a) urgencia, que se } \\
\text { refiere a la tendencia de vivir impulsos fuertes bajo con- } \\
\text { diciones de estímulos negativos, (b) premeditación como } \\
\text { tendencia a pensar y reflexionar sobre las consecuencias } \\
\text { de actuar y (c) perseverancia, implica la habilidad de } \\
\text { mantenerse en una tarea a pesar del aburrimiento o difi- } \\
\text { cultad. }\end{array}$ & (Whiteside \& Lynam , 2003) & 8 \\
\hline $\begin{array}{l}\text { Se divide en dos factores: (a) conductual o motor y (b) } \\
\text { cognitivo. (a) Se refiere a la inquietud motora, acciones } \\
\text { rápidas y (b) a la falta de planificación y previsión de la } \\
\text { conducta. }\end{array}$ & (Gerbing, Ahadi, \& Patton, 1987) & 7 \\
\hline $\begin{array}{l}\text { No pensar en las consecuencias, actuar sin pensar, falta } \\
\text { de evaluación de las consecuencias, no considerar las } \\
\text { consecuencias lógicas de las acciones, no planear. }\end{array}$ & $\begin{array}{l}\text { (Anderman, Cupp, \& Lane, 2009; Bar- } \\
\text { ker, Trentacosta, \& Salekin, 2011; Brook } \\
\text { \& Boaz, 2005; Castellani \& Rugle, 1995; } \\
\text { Gerbing et al., 1987; Smith, 1952) }\end{array}$ & 9 \\
\hline $\begin{array}{l}\text { Tomar decisiones rápidamente, responder prematura- } \\
\text { mente, actuar sin pensar, realizar conductas de riesgo. }\end{array}$ & $\begin{array}{l}\text { (Dougherty et al., 2003; Ghahremani et } \\
\text { al., 2013; Kaltiala-Heino et al., 2003; Zuc- } \\
\text { kerman, 1996) }\end{array}$ & 7 \\
\hline $\begin{array}{l}\text { Tres dimensiones: toma de decisiones, falta de atención y } \\
\text { falta de inhibición. }\end{array}$ & (Reynolds et al., 2008) & 4 \\
\hline $\begin{array}{l}\text { Tres dimensiones: (a) iniciación de la respuesta de forma } \\
\text { espontánea sin planearla, (b) inhibición de la respuesta el } \\
\text { fracaso para detener acciones ya iniciadas y (c) sensibili- } \\
\text { dad a las consecuencias como no tolerar el retraso de las } \\
\text { recompensas. }\end{array}$ & (Dougherty et al., 2009) & 3 \\
\hline $\begin{array}{l}\text { Impulsividad funcional y disfuncional, ambas relaciona- } \\
\text { das con el estilo de procesamiento de información. La } \\
\text { primera se relaciona con una tendencia a tomar decisio- } \\
\text { nes rápidas cuando son requeridas por la situación y se } \\
\text { obtiene un beneficio propio. La segunda se relaciona con } \\
\text { decisiones rápidas e irreflexivas que tienen consecuencias } \\
\text { negativas para el individuo. }\end{array}$ & (Dickman, 1996) & 5 \\
\hline $\begin{array}{l}\text { Impulsividad y reflexividad como un estilo cognitivo } \\
\text { definido por el número de errores antes de elegir la al- } \\
\text { ternativa correcta y el tiempo que los individuos tardan } \\
\text { en generar su primera elección. Tendencia a responder } \\
\text { rápidamente sin reflexionar. Se relaciona con la toma de } \\
\text { decisiones y solución de problemas donde se deben consi- } \\
\text { derar diferentes alternativas y valorar cuidadosamente las } \\
\text { consecuencias. }\end{array}$ & $\begin{array}{l}\text { (Donohew et al., 2000; Dougherty et al., } \\
\text { 2013; D’Zurilla, Chang, \& Sanna, 2003; } \\
\text { Kagan, Rosman, Day, Albert, \& Phillips, } \\
\text { 1964; Keller \& Ripoll, 2004; Oas, 1984; } \\
\text { Plutchik \& Van Praag, 1995; Zern, Ken- } \\
\text { ney, \& Kvaraceus, 1977) }\end{array}$ & 12 \\
\hline $\begin{array}{l}\text { Una predisposición para generar reacciones no planeadas } \\
\text { y rápidas frente a estímulos internos o externos }\end{array}$ & $\begin{array}{l}\text { (Leeman, Hoff, Krishnan-Sarin, Patock- } \\
\text { Peckham, \& Potenza, 2014) }\end{array}$ & 3 \\
\hline Total de artículos & & 63 \\
\hline
\end{tabular}

Fuente: elaboración propia 
TABLA 3

Resumen de instrumentos de evaluación

\begin{tabular}{|c|c|c|c|}
\hline $\begin{array}{l}\text { Instrumento de } \\
\text { evaluación }\end{array}$ & Descripción del instrumento & $\begin{array}{l}\text { Media alfa } \\
\text { reportada }\end{array}$ & $\begin{array}{l}\text { Número artículos } \\
\text { que la reportan }\end{array}$ \\
\hline $\begin{array}{l}\text { The Barratt } \\
\text { Impulsiveness } \\
\text { Scale (E) }\end{array}$ & $\begin{array}{l}\text { Consta de tres subescalas: impulsividad cognitiva, } \\
\text { impulsividad motora e impulsividad no planeada. }\end{array}$ & 0.8 & 30 \\
\hline $\begin{array}{l}\text { The Eysenck } \\
\text { Impulsiveness } \\
\text { Questionnaire } \\
\text { (E) }\end{array}$ & $\begin{array}{l}\text { Diseñado para evaluar los rasgos de personalidad con } \\
\text { tres subescalas: impulsividad, atrevimiento y empatía. Se } \\
\text { presume que la impulsividad y atrevimiento contribuyen a } \\
\text { las preferencias de riesgo. }\end{array}$ & 0.8 & 10 \\
\hline $\begin{array}{l}\text { The Matching } \\
\text { Familiar Figures } \\
\text { Test (B) }\end{array}$ & $\begin{array}{l}\text { Evalúa las dimensiones de reflexividad e impulsividad por } \\
\text { medio de la latencia y número de errores de las respuestas. }\end{array}$ & $\begin{aligned} \text { Errores } & =0.68 \\
\text { Latencia } & =0.93\end{aligned}$ & 10 \\
\hline $\begin{array}{l}\text { UPPS Impulsive } \\
\text { Behavior Scale } \\
\text { (E) }\end{array}$ & $\begin{array}{l}\text { Mide impulsividad por medio de las cinco dimensiones } \\
\text { del modelo de personalidad de los Cinco Factores por } \\
\text { medio de cuatro subescalas: Falta de premeditación, } \\
\text { urgencia, búsqueda de sensaciones y falta de } \\
\text { perseverancia. }\end{array}$ & 0.8 & 8 \\
\hline $\begin{array}{l}\text { Immediate } \\
\text { and Delayed } \\
\text { Memory Tasks } \\
\text { (IMT/DMT) } \\
\text { (B) }\end{array}$ & $\begin{array}{l}\text { Es una prueba de rendimiento que evalúa la impulsividad } \\
\text { como respuesta de iniciación o respuesta rápida por } \\
\text { medio de la comisión de errores. }\end{array}$ & No se reporta & 7 \\
\hline $\begin{array}{l}\text { Escala de } \\
\text { Impulsividad de } \\
\text { Plutchik (E) }\end{array}$ & $\begin{array}{l}\text { Tiene en cuenta la tendencia de involucrarse en } \\
\text { conductas compulsivas que reflejan pérdidas de control. } \\
\text { Aborda temas como: planeación, comportamientos } \\
\text { impulsivos, alimentación, control emocional y control } \\
\text { conductual. }\end{array}$ & 0.73 & 6 \\
\hline $\begin{array}{l}\text { GoStop Task } \\
\text { (B) }\end{array}$ & $\begin{array}{l}\text { Evalúa la inhibición de respuestas por medio de las claves } \\
\text { "go" y "stop". Las medidas principales, son el porcentaje } \\
\text { de respuestas inhibidas, es decir donde no se produce } \\
\text { ninguna respuesta y la latencia de respuesta. }\end{array}$ & No se reporta & 5 \\
\hline $\begin{array}{l}\text { Two Choice } \\
\text { Impulsivity } \\
\text { Paradigm (B) }\end{array}$ & $\begin{array}{l}\text { Valora la sensibilidad a las consecuencias teniendo en } \\
\text { cuenta que genera recompensas más grandes por demoras } \\
\text { largas y recompensas más pequeñas por demoras cortas. }\end{array}$ & No se reporta & 3 \\
\hline $\begin{array}{l}\text { Conners } \\
\text { Continuous } \\
\text { Performance } \\
\text { Test (B) }\end{array}$ & $\begin{array}{l}\text { Aprecia los problemas relacionados con atención y } \\
\text { contempla diferentes áreas como: falta de atención, } \\
\text { atención sostenida, vigilancia e impulsividad. Esta última } \\
\text { implica medir la velocidad de respuestas, respuestas } \\
\text { anticipadas y respuestas incorrectas. }\end{array}$ & No se reporta & 3 \\
\hline $\begin{array}{l}\text { Zuckerman- } \\
\text { Kuhlman } \\
\text { Personality } \\
\text { Questionnaire } \\
\text { (E) }\end{array}$ & $\begin{array}{l}\text { Diseñado para evaluar diferentes rasgos de la } \\
\text { personalidad relacionados con preferencias de conductas } \\
\text { de riesgo. Comprende cinco subescalas: sociabilidad, } \\
\text { ansiedad y neuroticismo, impulsividad y búsqueda de } \\
\text { sensaciones, agresión y actividad. }\end{array}$ & No se reporta & 3 \\
\hline $\begin{array}{l}\text { Single Key } \\
\text { Impulsivity } \\
\text { Paradigm } \\
\text { (SKIP). (B) }\end{array}$ & $\begin{array}{l}\text { Tiene en cuenta la tasa y el patrón de respuestas } \\
\text { operantes para obtener una recompensa; los participantes } \\
\text { son libres de responder con la frecuencia que quieran } \\
\text { para obtener la recompensa, la cual está relacionada con } \\
\text { la demora de la respuesta. }\end{array}$ & No se reporta & 2 \\
\hline
\end{tabular}

Nota. $\mathrm{B}=$ instrumentos de evaluación conductual y $\mathrm{E}=$ cuestionarios.

Fuente: elaboración propia 
TABLA 4

Aspectos relacionados con impulsividad

\begin{tabular}{lccclcc}
\hline \multicolumn{1}{c}{ Aspecto } & $\begin{array}{c}\text { Número de } \\
\text { artículos }\end{array}$ & \multicolumn{1}{c}{ Aspecto } & $\begin{array}{c}\text { Número de } \\
\text { artículos }\end{array}$ & \multicolumn{1}{c}{ Aspecto } & $\begin{array}{c}\text { Número de } \\
\text { artículos }\end{array}$ \\
\hline Problemas de conducta & 13 & Depresión & 6 & Toma de decisiones & 4 \\
\hline Medidas cognitivas & 13 & Consumo de drogas & 6 & Sobrepeso & 4 \\
\hline Agresión & 10 & Rasgos de personalidad & 5 & Déficit de atención & 3 \\
\hline $\begin{array}{l}\text { Características familiares } \\
\text { y contextuales }\end{array}$ & 9 & Consumo de alcohol & 5 & Rendimiento académico & 3 \\
\hline $\begin{array}{l}\text { Riesgo suicida } \\
\begin{array}{l}\text { Trastorno alimentario } \\
\text { alimenticio }\end{array}\end{array}$ & 7 & Ansiedad & Consumo de cigarrillos & 4 & Conductas sexuales & 2 \\
\hline
\end{tabular}

Fuente: elaboración propia.

definiciones de impulsividad, relacionando las medidas usadas y puntualizando los diferentes rasgos psicológicos con las cuales se relaciona en las investigaciones. El crecimiento de interés sobre este tema se hizo evidente en la revisión de la producción realizada sobre el estudio de impulsividad en adolescentes. Así, en la base de datos de SCOPUS, se evidencia que en el año 2000, 46 artículos abordan esta temática, mientras que en el 2014 aumentó a 304 artículos publicados.

Al analizar los 108 documentos incluidos en esta revisión sistemática bajo los criterios de inclusión establecidos, respecto a la conceptualización del constructo, se puede concluir: (a) es un constructo complejo que está atravesado por diferentes características y se puede abordar de diferentes formas. Cada definición se centra en características diferentes, de esta manera se puede definir la impulsividad como una forma funcional o disfuncional, motora o cognitiva, focalizándose en la actuación sin pensar, la toma de decisiones apresuradas, la falta de atención, la dificultad en el procesamiento de información e inhibición de la conducta, la reflexividad e impulsividad, etc.; (b) a pesar de ser un constructo complejo y multidimensional, no todos los estudios se ubican dentro de una definición clara, no la definen o la abordan desde diferentes conceptualizaciones; (c) no todas las definiciones implican establecer dimensiones, alguna de ellas la asumen como un constructo unidimensional y (d) la definición más común es la de asumir la impulsividad como un estilo cognitivo continuo que va de reflexividad a impulsividad.

En relación con la evaluación de la impulsividad, se resalta la gran variedad de instrumentos construidos, algunos de ellos son instrumentos "clásicos", como: The Barratt Impulsiveness Scale, The Eysenck Impulsiveness Questionnaire y The Matching Familiar Figures Test. Igualmente, se tiene en cuenta que los resultados confirman que instrumentos de evaluación de impulsividad se encuentran clasificados en medidas como cuestionarios o medidas de evaluación conductual (Carver \& White, 1994; Derryberry \& Reed, 2002; Glenn \& Klonsky, 2010; Khurana et al., 2012; Rachlin, 2004; Richards et al., 1999). En los artículos revisados se destacan 14 instrumentos como cuestionarios y 15 como instrumentos de evaluación conductual.

Respecto a la relación de impulsividad con otros constructos, se identificó que se tiende a relacionar el concepto más con problemas de conducta, medidas cognitivas, agresión, aspectos familiares y contextuales y riesgo suicida. Se identificó que la impulsividad ha sido menos explorada con aspectos como conductas de riesgo sexual, diferencias de sexo, rendimiento académico, sobrepeso, toma de decisiones, regulación emocional y problemas de sueño. En este sentido, se recomienda para 
futuras investigaciones abordar el estudio de la relación de estos constructos con la impulsividad en adolescentes y, de esta manera, contribuir a la ampliación de esta área de estudio, tal como lo demuestran las investigaciones realizadas por Sobral, Villar, Gómez-Fraguela, Romero y Luengo (2013) y Haghighi, Ghanavati y Rahimi (2015), quienes abordan temáticas innovadoras en relación con la impulsividad.

La recopilación de la conceptualización y los instrumentos usados para evaluar impulsividad en adolescentes se lleva a cabo para elaborar una guía que permita tomar decisiones a los futuros investigadores que estén interesados en realizar estudios de evaluación sobre el tema. Es importante tener en cuenta para futuros estudios que para poder evaluar este constructo, es necesario definir si se empleará un instrumento de evaluación conductual o un cuestionario. Esto es relevante ya que se tiene en cuenta que las investigaciones ponen de manifiesto correlaciones bajas no significativas cuando se comparan estas dos formas de evaluación (Dougherty et al., 2009; Heyman \& Gibb, 2006; Krishnan-Sarin et al., 2007; Thompson et al., 2006; Wittmann, Arce, \& Santisteban, 2008), por lo cual se recomienda que en la comparación se asegure que los componentes evaluados sean parecidos, para poder brindar mayor claridad frente a la relación entre ellos y unificación de las diferentes definiciones del constructo. Por otra parte, se recomienda que se identifiquen y reporten los coeficientes alfa de los instrumentos usados, para poder establecer cómo se están comportando estos instrumentos en población adolescente.

Resulta curioso que no encaje la definición más usada, la de reflexividad-impulsividad, con el instrumento más usado, The Barrat Impulsivity Scale, lo que puede deberse a dos razones: primero, que muchos artículos no eran claros en cuanto a la definición del constructo y segundo, muchos usaban varias medidas de evaluación, mezclando varios cuestionarios y alguna medida de evaluación conductual. Estas dificultades también han sido identificadas en otras revisiones, donde se argumenta que los estudios consultados son dispares en el enfoque y el análisis de los resultados es limitado por los inconvenientes en la presentación de los resultados incompletos de las investigaciones (Zwi, Jones, Thorgaard, York, \& Dennis, 2011).

\section{Referencias}

Anderman, E. M., Cupp, P. K., \& Lane, D. (2009). Impulsivity and academic cheating. The Journal of Experimental Education, 78(1), 135-150. doi: 10.1080/00220970903224636

Bari, A., \& Robbins, T. W. (2013). Inhibition and impulsivity: Behavioral and neural basis of response control. Progress in Neurobiology, 108, 44-79. doi:10.1016/j.pneurobio.2013.06.005

Barker, E. D., Trentacosta, C. J., \& Salekin, R. T. (2011). Are impulsive adolescents differentially influenced by the good and bad of neighborhood and family? Journal of Abnormal Psychology, 120(4), 981-986. doi:10.1037/a0022878

Barratt, E. S. (1994). Impulsiveness and aggression. En J. A. Monahan \& H. J. Steadman (Eds.), Violence and mental disorder: Developments in risk assessment (pp. 61-79). Chicago: University of Chicago.

Bjørkly, S. (2013). A systematic review of the relationship between impulsivity and violence in persons with psychosis: Evidence or spin cycle? Aggression and Violent Behavior, 18, 753-760. doi:10.1016/j. avb.2013.08.001

Brook, U., \& Boaz, M. (2005). Impulsivity among adolescents with ADHD and bronchial asthma. The Indian Journal of Pediatrics, 72(11), 939-942. doi: 10.1007/BF02731668

Buela-Casal, G., Carretero-Dios, H., \& Santos-Roig, M. (2001). Consistencia longitudinal de la reflexividad-impulsividad evaluada por el Matching Familiar Figures Test-20 (MFFT 20). Clínica y Salud, 12(1), 51-70.

Buela-Casal, G., Carretero-Dios, H., Santos-Roig, M., \& Bermúdez, M. P. (2003). Psychometric properties of a Spanish adaptation of the Matching Familiar Figures Test. European Journal of Psychological Assesment, 19(2), 151-159. doi:10.1027//10155759.19.2.151

Buelow, M. T., \& Suhr, J. A. (2009). Construct validity of the Iowa Gambling Task. Neuropsychology Review, 19(1), 102-114. doi:10.1007/s11065-009-9083.4 
Cabral, P. (2006). Attention deficit disorders: Are we barking up the wrong tree? European Journal of Paediatric Neurology, 10(2), 66-77. doi:10.1016/j. ejpn.2006.02.004

Carrillo-De-La-Peña, M. T., Otero, J. M., \& Romero, E. (1993). Comparison among various methods of assessment of impulsiveness. Perceptual and Motor Skills, 77(2), 567-575. doi:10.2466/ pms.1993.77.2.567

Carver, C. S., \& White, T. L. (1994). Behavioral inhibition, behavioral activation, and affective responses to impending reward and punishment. The BIS/ BAS scales. Journal of Personality and Social Psychology, 67(2), 319-333. doi:10.1037/0022-3514.67.2.319

Castellani, M. A., \& Rugle, L. (1995). A comparison of pathological gamblers to alcoholics and cocaine misusers on impulsivity, sensation seeking, and craving. International Journal of Addictions, 30(3), 275-289. doi:10.3109/10826089509048726

Claes, L., Vertommen, H., \& Braspenning, N. (2000). Psychometric properties of the Dickman impulsivity inventory. Personality and Individual Differences, 29(1), 27-35. doi:10.1016/S0191-8869(99)00172-5

Coskunpinar, A., \& Cyders, M. A. (2013). Impulsivity and substance-related attentional bias: A meta-analytic review. Drug and Alcohol Dependence, 133(1), 1-14. doi:10.1016/j.drugalcdep.2013.05.008

Derryberry, D., \& Reed, M. A. (2002). Anxiety-related attentional biases and their regulation by attentional control. Journal of Abnormal Psychology, 111(2), 225-236. doi:10.1037/0021-843X.111.2.225

DeWit, H. (2009). Impulsivity as a determinant and consequence of drug use: A review of underlying processes. Addiction Biology, 14(1), 22-31. doi:10.1111/ j.1369-1600.2008.00129.x

Dickman, S. J. (1990). Functional and dysfunctional impulsivity: Personality and cognitive correlates. Journal of Personality and Social Psychology, 58(1), 95-102. doi:10.1037/0022-3514.58.1.95

Donohew, L., Zimmerman, R., Cupp, P. S., Novak, S., Colon, S., \& Abell, R. (2000). Sensation seeking, impulsive decision-making and risky sex: Implications for risk-taking and design of interventions. Personality and Individual Differences, 28(6), 1079. 1091. doi:10.1016/S0191-8869 (99)00158-0
Dougherty, D. M., Bjork, J. M., Harper, R. A., Marsh, D. M., Moeller, F. G., Mathias, C. W., \& Swann, A. C. (2003). Behavioral impulsivity paradigms: A comparison in hospitalized adolescents with disruptive behavior disorders. Journal of Child Psychology and Psychiatry, 44(8), 1145-1157. doi:10.1111/1469. 7610.00197

Dougherty, D. M., Mathias, C. W., Dawes, M. A., Furr, R. M., Charles, N. E., Liguori, A., ... Acheson, A. (2013). Impulsivity, attention, memory, and decision-making among adolescent marijuana users. Psychopharmacology, 226(2), 307-319. doi:10.1007/s00213-012-2908-5

Dougherty, D. M., Mathias, C. W., Marsh-Richard, D. M., Furr, R. M., Nouvion, S. O., \& Dawes, M. A. (2009). Distinctions in behavioral impulsivity: Implications for substance abuse research. Addictive Disorders $\&$ their Treatment, 8(2), 61-73. doi:10.1097/ ADT.0b013e318172e488

D’Zurilla, T. J., Chang, E. C., \& Sanna, L. J. (2003). Selfesteem and social problem solving as predictors of aggression in college students. Journal of Social and Clinical Psychology, 22, 424-440. doi:10.1521/ jscp.22.4.424.22897

Fields, S. A., Sabet, M., \& Reynolds, B. (2013). Dimensions of impulsive behavior in obese, overweight, and healthy-weight adolescents. Appetite, 70, 60 66. doi:10.1016/j.appet.2013.06.089

Fischer, S., Smith, G. T., \& Cyders, M. A. (2008). Another look at impulsivity: A meta-analytic review comparing specific dispositions to rash action in their relationship to bulimic symptoms. Clinical Psychology Review, 28(8), 1413-1425. doi:10.1016/j. cpr.2008.09.001

Gerbing, D. W., Ahadi, S. A., \& Patton, J. H. (1987). Towards a conceptualization of impulsivity: Components across the behavioural and self-report domains. Multivariate Behavioural Research, 22, 357-379. doi:10.1207/s15327906mbr2203 6

Ghahremani, D. G., Oh, E. Y., Dean, A. C., Mouzakis, K., Wilson, K. D., \& London, E. D. (2013). Effects of the youth empowerment seminar on impulsive behavior in adolescents. Journal of Adolescent Health, 53(1), 139-141. doi:10.1016/j.jadohealth.2013.02.010 
Gilbert, K. E., Kalmar, J. H., Womer, F. Y., Markovich, P. J., Pittman, B., Nolen $\square$ Hoeksema, S., \& Blumberg, H. P. (2011). Impulsivity in adolescent bipolar disorder. Acta Neuropsychiatrica, 23(2), 57-61. doi:10.1111/j.1601-5215.2011.00522.x

Glenn, C. R., \& Klonsky, E. D. (2010). A multimethod analysis of impulsivity in nonsuicidal self-injury. Personality Disorders: Theory, Research, and Treatment, 1(1), 67-75. doi:10.1037/a0017427

Gorlyn, M., Keilp, J. G., Tryon, W. W., \& Mann, J. J. (2005). Performance test correlates of component factors of impulsiveness. Personality and Individual Differences, 38, 1549-1559. doi:10.1016/j. paid.2004.09.014

Grant, J. E., \& Chamberlain, S. R. (2014). Impulsive action and impulsive choice across substance and behavioral addictions: Cause or consequence? Addictive Behaviors, 39(11), 1632-1639. doi:10.1016/j. addbeh.2014.04.022

Gullo, M. J., \& Dawe, S. (2008). Impulsivity and adolescent substance use: Rashly dismissed as "allbad"? Neuroscience 83 Biobehavioral Reviews, 32, 1507 1518. doi:10.1016/j.neubiorev.2008.06.003

Haghighi, M., Ghanavati, M., \& Rahimi, A. (2015). The role of gender differences in the cognitive style of impulsivity/reflectivity and EFL. Success.ProcediaSocial and Behavioral Sciences, 192, 467-474.

Heyman, G., \& Gibb, S. (2006). Delay discounting in college cigarette chippers. Behavioural Pharmacology, 17(8), 669-679. doi:10.1097/FBP.0b013e3280116cfe

Janssen, T., Larsen, H., Peeters, M., Boendermaker, W. J., Vollebergh, W. A., \& Wiers, R. W. (2015). Do online assessed self-report and behavioral measures of impulsivity-related constructs predict onset of substance use in adolescents? Addictive Behaviors Reports, 1, 12-18. doi: 10.1016/j.abrep.2015.01.002

Kagan, J., Rosman, B. L., Day, D., Albert, J., \& Phillips, W. (1964). Information processing in the child: Significance of analytic and reflective attitudes. Psychological Monographs: General and Applied, 78, 1-37. doi:10.1037/h0093830

Kaltiala-Heino, R., Rissanen, A., Rimpelä, M., \& Rantanen, P. A. I. (2003). Bulimia and impulsive behaviour in middle adolescence. Psychotherapy and Psychosomatics, 72(1), 26-33. doi:10.1159/000067187
Keller, J., \& Ripoll, H. (2004). Stability of reflective impulsive style in coincidence anticipation motor tasks. Learning and Individual Differences, 14(4), 209-218. doi:10.1016/j.lindif.2004.02.004

Khodarahimi, S. (2013). Impulsivity, aggression, and psychopathic deviation in a sample of Iranian adolescents and young adults: Gender differences and predictors. Journal of Forensic Psychology Practice, 13(5), 373-388. doi:10.1080/15228932.20 13.829733

Khurana, A., Romer, D., Betancourt, L. M., Brodsky, N. L., Giannetta, J. M., \& Hurt, H. (2012). Early adolescent sexual debut: The mediating role of working memory ability, sensation seeking, and impulsivity. Developmental Psychology, 48(5), 14161428. doi:10.1037/a0027491

Krishnan-Sarin, S., Reynolds, B., Duhig, A. M., Smith, A., Liss, T., McFetridge, A., ... Potenza, M. N. (2007). Behavioral impulsivity predicts treatment outcome in a smoking cessation program for adolescent smokers. Drug and Alcohol Dependence, 88(1), 79-82. doi:10.1016/j.drugalcdep.2006.09.006

Leeman, R. F., Hoff, R. A., Krishnan-Sarin, S., PatockPeckham, J. A., \& Potenza, M. N. (2014). Impulsivity, sensation-seeking, and part-time job status in relation to substance use and gambling in adolescents. Journal of Adolescent Health, 54(4), 460-466. doi:10.1016/j.jadohealth.2013.09.014

Leshem, R., \& Glicksohn, J. (2012). A critical look at the relationship between impulsivity and decisionmaking in adolescents: are they related or separate factors? Developmental Neuropsychology, 37(8), 712 731. doi:10.1080/87565641.2012.718815

Letzring, T. D., Block, J., \& Funder, D. C. (2005). Ego-control and ego-resiliency: generalization on self-report scales based on personality descriptions from acquaintances, clinicians, and the self. Journal of Research in Personality, 39(4), 395-422. doi:10.1016/j.jpp.2004.06.003

Martínez-Loredo, V., Fernández-Hermida, J. R., Fernández-Artamendi, S., Carballo, J. L., \& GarcíaRodríguez, O. (2015). Spanish adaptation and validation of the Barratt Impulsiveness Scale for early adolescents (BIS-11-A). International Journal of Clinical and Health Psychology. Publicación anticipada en línea. doi: 10.1016/j.ijchp.2015.07.002 
Mathias, C. W., Marsh-Richard, D. M., \& Dougherty, D. M. (2008). Behavioral measures of impulsivity and the law. Behavioral Sciences 83 the Law, 26(6), 691-707. doi:10.1002/bsl.841

Najt, P., Pérez, J., Sánchez, M., Peluso, M. A. M., Glahn, D., \& Soares, J. C. (2007). Impulsivity and bipolar disorder. European Neuropsychopharmacology, 17(5), 313-320. doi:10.1016/j.euroneuro.2006.10.002

Oas, P. (1984). Validity of a Draw-A-Person and Bender Gestalt tests as measures of impulsivity with adolescents. Journal of Consulting and Clinical Psychology, 52(6), 1011-1019. doi:10.1037/0022006X.52.6.1011

Ouzir, M. (2013). Impulsivity in schizophrenia: A comprehensive update. Aggression and Violent Behavior, 18(2), 247-254. doi:10.1016/j.avb.2012.11.014

Patton, J. H., Stanford, M. S., \& Barratt, E. S. (1995). Factor structure of the Barratt Impulsiveness Scale. Journal of Clinical Psychology, 51(6), 768-774.

Perestelo-Pérez, L. (2013). Standards on how to develop and report systematic reviews in psychology and health. International Journal of Clinical and Health Psychology, 13(1), 49-57. doi:10.1016/S16972600(13)70007-3

Plutchik, R., \& Van Praag, H. M. (1995). The nature of impulsivity definitions, ontology, genetics and relations to aggression. New York: Wiley.

Poythress, N. G., \& Hall, J. R. (2011). Psychopathy and impulsivity reconsidered. Aggression and Violent Behavior, 16(2), 120-134. doi:10.1016/j. avb.2011.02.003

Rachlin, H. (2004). The science of self-control. Cambridge, MA: Harvard University Press.

Richards, J. B., Zhang, L., Mitchell, S. H., \& de Wit, H. (1999). Delay or probability discounting in a model of impulsive behavior. Effect of alcohol. Journal of Experimental Analysis of Behavior, 71(2), 121-143. doi: 10.1901/jeab.1999.71-121

Reynolds, B., Ortengren, A., Richards, J. B., \& de Wit, H. (2006). Dimensions of impulsive behavior: Personality and behavioral measures. Personality and Individual Differences, 40(2), 305-315. doi:10.1016/j. paid.2005.03.024

Reynolds, B., Penfold, R. B., \& Patak, M. (2008). Dimensions of impulsive behavior in adolescents:
Laboratory behavioral assessments. Experimental and Clinical Psychopharmacology, 16(2), 124-131. doi:10.1037/1064-1297.16.2.124

Romer, D. (2010). Adolescent risk taking, impulsivity, and brain development: Implications for prevention. Developmental Psychobiology, 52(3), 263-276. doi:10.1002/dev.20442

Saddichha, S., \& Schuetz, C. (2014). Impulsivity in remitted depression: A meta-analytical review. Asian Journal of Psychiatry, 9, 13-16. doi:10.1016/j. ajp.2014.02.003

Smith, L. (1952). A dictionary of psychiatry for the Layman. London: Maxwell.

Sobral, J., Villar, P., Gómez-Fraguela, J. A., Romero, E., \& Luengo, M. A. (2013). Interactive effects of personality and separation as acculturation style on adolescent antisocial behaviour. International Journal of Clinical and Health Psychology, 13(1), 25-31.

Thompson, L. L., Whitmore, E. A., Raymond, K. M., \& Crowley, T. J. (2006). Measuring impulsivity in adolescents with serious substance and conduct problems. Assessment, 13(1), 3-15. doi: $10.1177 / 1073191105282247$

Whiteside, S. P., \& Lynam, D. R. (2001). The Five Factor Model and impulsivity: Using a structural model of personality to understand impulsivity. Personality and Individual Differences, 30(4), 669-689.

Whiteside, S. P., \& Lynam, D. R. (2003). Understanding the role of impulsivity and externalizing psychopathology in alcohol abuse: Application of the UPPS impulsive behavior scale. Experimental and Clinical Psychopharmacology, 11(3), 210-217. doi:10.1037/1064-1297.11.3.210

Winstanley, C. A., Eagle, D. M., \& Robbins, T. W. (2006). Behavioral models of impulsivity in relation to ADHD: Translation between clinical and preclinical studies. Clinical Psychology Review, 26(4), 379-395. doi:10.1016/j.cpr.2006.01.001

Wittmann, M., Arce, E., \& Santisteban, C. (2008). How impulsiveness, trait anger, and extracurricular activities might affect aggression in school children. Personality and Individual Differences, 45(7), 618. 623. doi:10.1016/j.paid.2008.07.001

Wittmann, M., \& Paulus, M. P. (2008). Decision making, impulsivity and time perception. Trends 
in Cognitive Sciences, 12(1), 7-12. doi:10.1016/j. tics.2007.10.004

Zern, D. S., Kenney, H. J., \& Kvaraceus, W. C. (1977). The cognitive style dimension of reflectivity-impulsivity in normal and emotionally disturbed adolescents. The Journal of Genetic Psychology, 131, 107-113. doi: 10.1080/00221325.1977.10533279

Zuckerman, M. (1996). The psychobiological model for impulsive unsocialized sensation seeking: A comparative approach. Neuropsychobiology, 34(3), 125-129. doi:10.1159/000119303

Zwi, M., Jones, H., Thorgaard, C., York, A., \& Dennis, J. A. (2011). Parent training interventions for Attention Deficit Hyperactivity Disorder (ADHD) in children aged 5 to 18 years. Cochrane Database of Systematic Reviews, 12, Art. ${ }^{\circ} \mathrm{CD}$ 003018. doi: 10.1002/14651858.CD003018.CD003018 
